\title{
Spectrally dependent photovoltages in Schottky photodiode based on (100) B-doped diamond
}

\author{
Jan Čermák, ${ }^{1, a)}$ Yasuo Koide, ${ }^{2}$ Daisuke Takeuchi, ${ }^{3}$ and Bohuslav Rezek ${ }^{1}$ \\ ${ }^{1}$ Institute of Physics, Academy of Sciences of the Czech Republic, Cukrovarnická 10, 16200 Prague 6, \\ Czech Republic \\ ${ }^{2}$ Sensor Materials Center, National Institute for Material Science (NIMS), 1-1 Namiki, Tsukuba 305-0044, \\ Japan \\ ${ }^{3}$ Energy Technology Research Institute, National Institute of Advanced Industrial Science and Technology \\ (AIST), 1-1-1 Umezono, Tsukuba 305-8568, Japan
}

(Received 17 June 2013; accepted 25 January 2014; published online 7 February 2014)

Spectrally and spatially resolved photovoltages were measured by Kelvin probe force microscopy (KPFM) on a Schottky photo-diode made of a $4 \mathrm{~nm}$ thin tungsten-carbide (WC) layer on a $500 \mathrm{~nm}$ oxygen-terminated boron-doped diamond epitaxial layer (O-BDD) that was grown on a Ib (100) diamond substrate. The diode was grounded by the sideways ohmic contact (Ti/WC), and the semitransparent Schottky contact was let unconnected. The electrical potentials across the device were measured in dark (only $650 \mathrm{~nm}$ LED of KPFM being on), under broad-band white light (halogen lamp), UV (365 nm diode), and deep ultraviolet (deuterium lamp) illumination. Illumination induced shift of the electrical potential remains within $210 \mathrm{mV}$. We propose that the photovoltage actually corresponds to a shift of Fermi level inside the BDD channel and thereby explains orders of magnitude changes in photocurrent. @ 2014 AIP Publishing LLC.

[http://dx.doi.org/10.1063/1.4864420]

\section{INTRODUCTION}

Diamond is a material which is intensively studied for various electronic as well as opto-electronic applications. In addition to its hardness, high thermal conductivity, and chemical resistivity, it exhibits an optical band-gap of $5.5 \mathrm{eV}$. Thus, it is optically transparent in the visible part of optical spectrum, and it absorbs deep ultraviolet (DUV) light. Therefore, a diamond-based DUV light-emitting diodes $^{1}$ or photodetectors ${ }^{2,3}$ are some of the highly perspective applications of diamond. For example, photodetectors designed as a p-i-n or a metal-semiconductormetal structures have already been developed and even applied in a solar DUV irradiance monitoring project. ${ }^{4,5}$ Another approach is a construction of a Schottky photodiode, which is expected to exhibit lower leakage current, lower noise level, zero-bias operation, and higher quantum efficiency. ${ }^{2}$

Promising concept of a Schottky photodiode based DUV photodetector seems to be a semitransparent (around $50 \%$ for wavelengths between 200 and $300 \mathrm{~nm}$ ) planar metal contact fabricated on an oxidized boron-doped epitaxial diamond layer (O-BDD) on an intrinsic high-pressure high-temperature diamond substrate. ${ }^{2}$ Suitable metal for the Schottky contact is, for example, tungsten carbide (WC), since it is thermally stable, oxidation-resistive highly electrically conductive material which is nonreactive with diamond at high temperatures. ${ }^{6}$ Such photodiode exhibits photocurrent gain and a respectable ratio of $10^{6}$ between the response to DUV and visible light at a reverse bias voltage as small as $2 \mathrm{~V}^{7}$ The response to illumination has been explained in terms of

${ }^{\text {a)} E l e c t r o n i c ~ m a i l: ~ c e r m a k j @ f z u . c z ~}$ boron-induced deep defects and the spatially charged nitrogen-related traps in the $\mathrm{Ib}$ diamond substrate. ${ }^{8}$ This mechanism has been deduced indirectly based on photocurrent measurements.

More direct insight into photo-response and energetic configuration can be obtained by measurement of photovoltages. For this purpose, Kelvin probe force microscopy (KPFM) is most suitable as it enables local characterization of microscopic devices directly with respect to a reference (typically a grounded Ohmic contact). For instance, KPFM was used to identify redistribution of charge carriers in a system consisting of intrinsic diamond and an organic dye (polypyrrole). The changes of surface potential in the dark and under illumination were explained by a transfer of charge carriers (holes) from the dye to diamond, and energetic model of the system has been proposed. ${ }^{9}$ The KPFM actually characterized the photovoltage effects in the polypyrrolediamond junction which was buried several tens of nm below the polypyrrole surface.

In this work, we employ KPFM to gain deeper insight into electronic configuration of the DUV-sensitive diamond Schottky photodiode and its function under various illumination. Illumination by light of various wavelengths can be used to select a specific type of photovoltage generation mechanisms. Therefore, by using KPFM, we study changes of electrical potential at the important parts of the diamond Schottky photo-diode under white light, UV, and DUV illumination. Unlike photocurrent measurements, the photovoltages are obtained under static conditions in non-biased device with a floating Schottky contact. Using such data, we elaborate a model of the device function and discuss the correlation of photovoltages and photocurrents as well as the role of the Ib substrate. 


\section{EXPERIMENTAL}

The Schottky photo-diode has been fabricated on a high-pressure high-temperature Ib (100) diamond crystal substrate. O-BDD layer in the thickness of around $500 \mathrm{~nm}$ was grown using microwave plasma-enhanced chemical vapor deposition from $\mathrm{CH}_{4}(0.4 \mathrm{ppm})$ to $\mathrm{H}_{2}(500 \mathrm{ppm})$ as source gases in an apparatus commonly used for growth of boron-doped diamond layers. The residual boron atoms from the deposition chamber doped the layer to an estimated concentration of $10^{15} \mathrm{~cm}^{-3}$ or lower. Oxidization of the surface was performed by dipping the epilayer in a boiling 1:1 acid solution of $\mathrm{H}_{2} \mathrm{SO}_{4} / \mathrm{HNO}_{3}$. The Schottky junction was prepared by depositing a $4 \mathrm{~nm}$ thin semitransparent WC contact. Ohmic contact was made sideways by a Ti layer covered by a WC layer as a protection. The detailed description of the preparation process can be found in the literature (unintentional doping). ${ }^{8}$

The microscopic characterization was performed by an NTEGRA (NT-MDT) AFM system. Pt/Cr coated cantilevers with nominal resonance frequency of $75 \mathrm{kHz}$ were used for KPFM. The Ohmic Ti/WC contact was grounded and the Schottky WC contact was floating. During the KPFM measurements, the device was illuminated by three types of light sources: AFM internal halogen lamp (broad-band visible light, co-axial), a UV light-emitting diode (maximum at $365 \mathrm{~nm}$ ), and a deuterium DUV lamp. UV and DUV illumination was coupled via a quartz optical fibre which was placed with a slight angle $\left(\sim 30^{\circ}\right)$ with respect to the sample normal to avoid screening by AFM tip. The photo-KPFM measurement setup scheme and photograph are shown in Fig. 1.

The slow scan axis was disabled during the KPFM experiment. Thus, the AFM tip scanned the same topographic line profile repeatedly. This method protects the KPFM data from topographical artifacts. The first parts (bottom) of the images were scanned in the dark, second (middle) under illumination, and third (top) in the dark again to confirm reproducibility, no influence of the tip, or measurement instability.

For construction of energetic schemes, we obtained electron affinity of O-BDD surface and work function of metallic contacts by total photoyield spectroscopy (TPYS). ${ }^{10}$ In TPYS, the quantum efficiency of photoelectron emission is measured as a function of photon energy. TPYS was carried out with $\mathrm{Xe}$ and $\mathrm{D}_{2}$ lamps. This provides applicable photoexcitation range from $2.0 \mathrm{eV}$ to $7.3 \mathrm{eV}$ on our samples. A double monochromator is used to disperse the light with a resolution of $15 \mathrm{meV}$ at $248 \mathrm{~nm}(h \nu=5 \mathrm{eV})$ using $200 \mu \mathrm{m}$ input/output slits. As TPYS measures the energy gap between occupied states and the vacuum level, the surface Fermi level is not affecting the results in a complex way as in X-ray (XPS) or ultra-violet (UPS) photoelectron emission spectroscopy. Electron affinity of diamond with various surface terminations can be thereby deduced in a straightforward way. ${ }^{11}$

Using Fowler's photoelectron emission theory, TPYS can be also used for metal work function calibration in natural (air adsorbed) condition which is essential for deducing
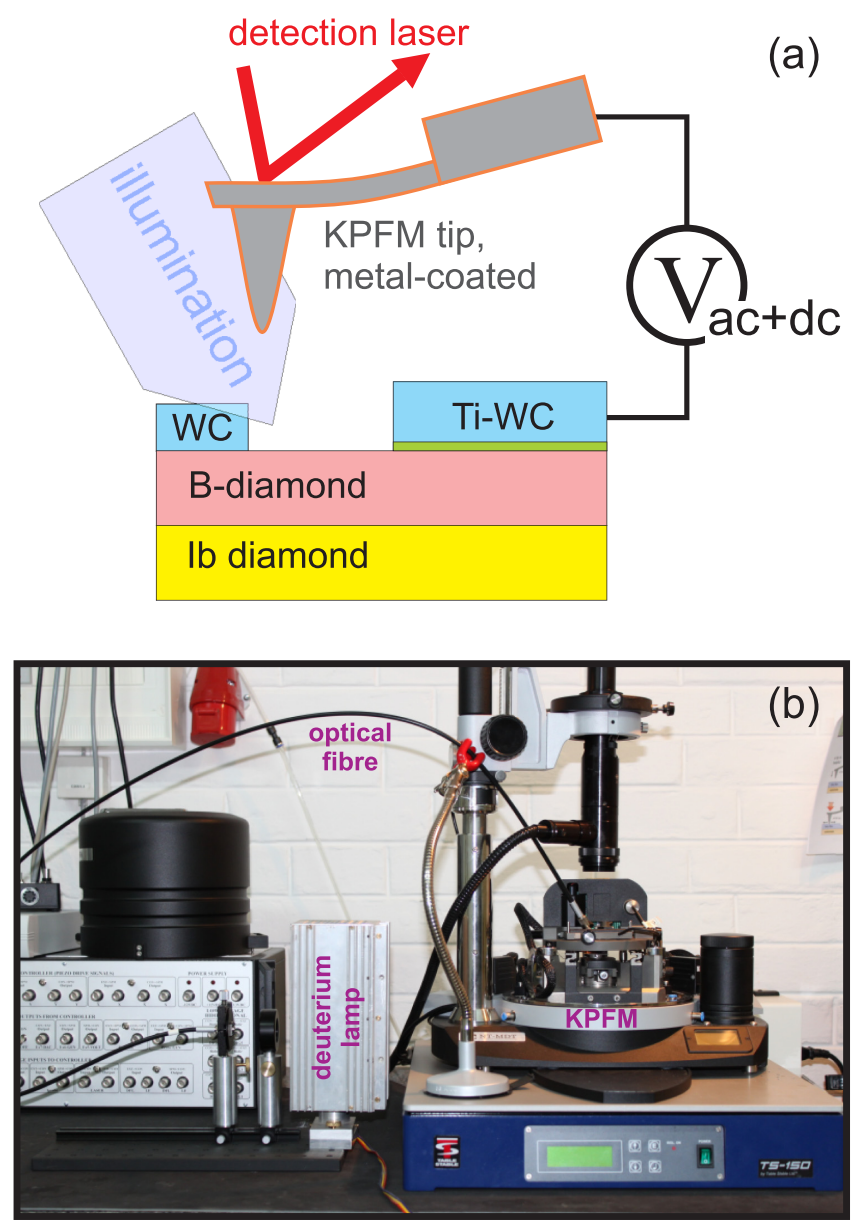

FIG. 1. (a) Schematic drawing of the experimental setup showing the sample structure, connection of the KPFM probe, and illumination. (b) Photo of the experimental setup showing the KPFM microscope and the deuterium lamp with optical fiber coupling.

diamond surface work function from KPFM data. ${ }^{12}$ Similar method for work function calibration is XPS/UPS; ${ }^{13}$ however, it cannot be used for evaluation of electron affinity.

TPYS thus represents a complementary tool to KPFM. Together, they can be used to construct a surface and subsurface energetic band configuration by using relatively straightforward guideline. The guideline scheme is shown in Fig. 2. Red labels denote values obtained directly from experiment, green labels denote parameters well known from literature, and blue labels denote deduced values. We can use TPYS to obtain work function of the reference metal. By adding potential difference obtained by KPFM across the sample and the reference metal, we deduce surface work function, i.e., position of the surface Fermi level below the vacuum level. By subtracting band gap width of the studied material, which is typically well know from the literature, from the ionization energy measured by TPYS, we obtain electron affinity of the material with specific surface treatment. At the same time, we get energetic position of the valence band maximum and conduction band minimum at the surface. When we combine all these energetic levels at the surface with the Fermi level position in the bulk, again typically well known from literature or measurable for instance by XPS, we can estimate the surface band bending direction 


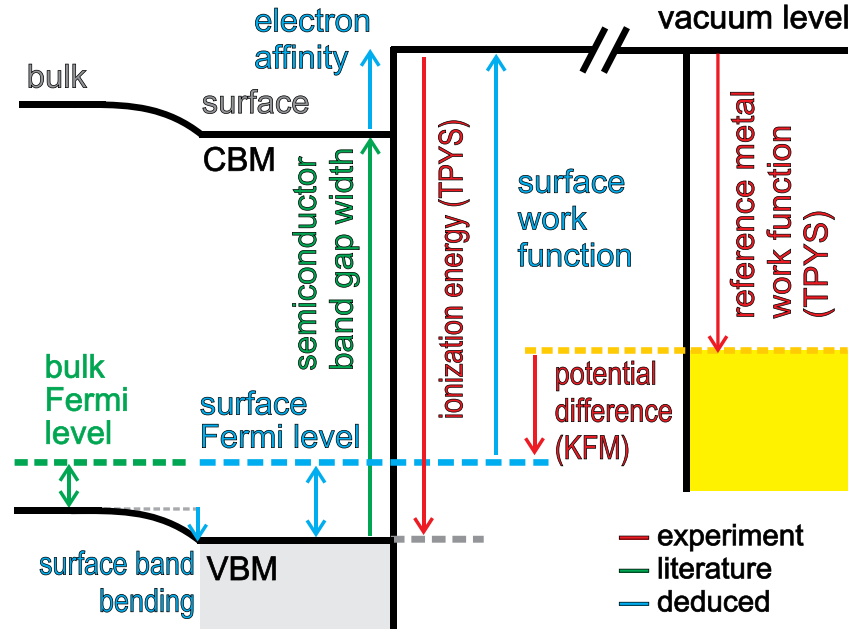

FIG. 2. Schematic guideline for construction of surface and sub-surface energetic band configurations based on KPFM and TPYS measurements. Red labels denote values obtained directly from experiment, green labels denote parameters well known from literature, and blue labels denote deduced values.

and magnitude. Thereby, we obtain the complete energetic configuration which can be used to describe and understand the material and device function.

\section{RESULTS}

Fig. 3 shows typical KPFM data measured in the dark and under illumination by the UV LED diode. In the dark, the potential difference between the grounded Ohmic contact and the O-BDD surface is about $400 \mathrm{mV}$. The potential difference between the Schottky contact and the exposed OBDD layer is about $600 \mathrm{mV}$. The potential on the grounded Ohmic Ti + WC contact remains at the same level both in the dark and under UV illumination. The potential on the exposed O-BDD surface shifts downwards by $\sim 110 \mathrm{mV}$ under the UV illumination. Higher shift $(150 \mathrm{mV})$ is observed on the WC Schottky contact. After the illumination was switched off, the potential returned to the same levels as prior to the illumination.
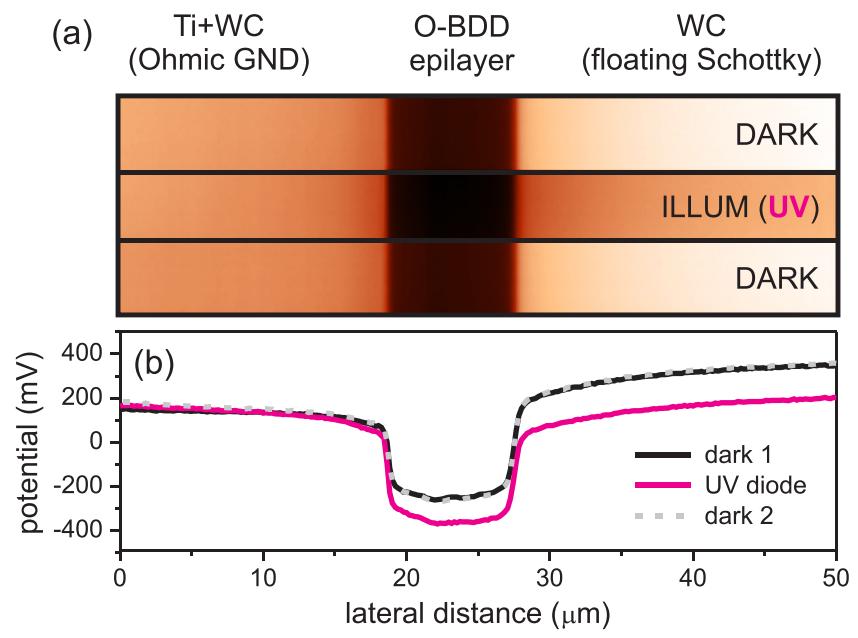

FIG. 3. (a) Image and (b) profiles of the KPFM potential across the diamond Schottky photodiode in the dark and under illumination by UV lightemitting diode.

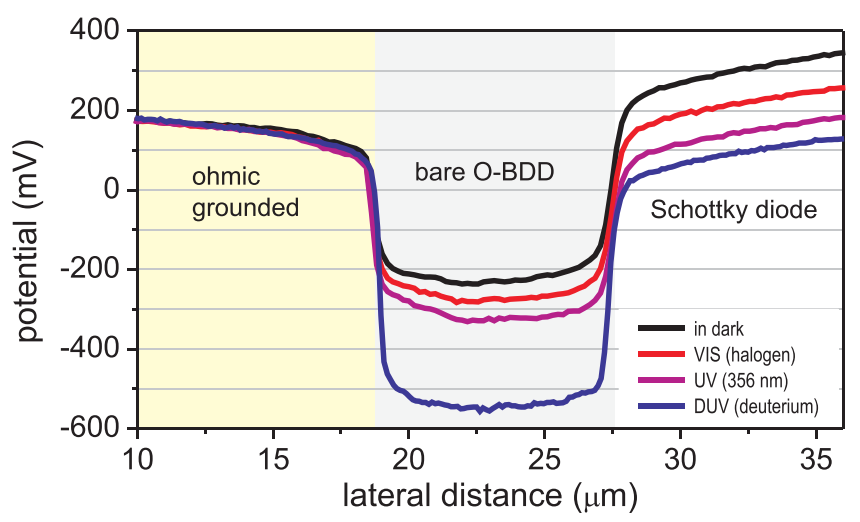

FIG. 4. KPFM potential profiles across the diamond Schottky photodiode in the dark and under illumination by the white light (halogen), UV diode $(365 \mathrm{~nm})$, and DUV (deuterium lamp). The potentials returned to dark state after the illumination was switched off except for DUV where it remained persistent.

Fig. 4 summarizes KPFM potential profiles measured in the dark and under illumination by the white light (halogen), UV diode (365 nm), and DUV (deuterium lamp). The potentials returned to dark state after the illumination was switched off except for DUV. After the DUV illumination was switched off, the photovoltage remained persistent for several days or even weeks if the resetting procedure ${ }^{8}$ was not applied. As the potential on the grounded Ohmic $\mathrm{Ti}+$ WC contact remains at the same level both in the dark and under any of the illuminations (see, e.g., Fig. 3), we used that as a reference to align the profiles on the potential (Y) axis.

The photovoltage on the Schottky contact increases to more negative values with decreasing illumination wavelength (i.e., increasing energy). The photovoltage is $-50 \mathrm{mV}$ under the white light, $-110 \mathrm{mV}$ under the UV diode, and $-210 \mathrm{mV}$ under the DUV illumination. For comparison, the photovoltage becomes increasingly more negative also on the exposed O-BDD layer: $-85 \mathrm{mV}$ under white light, $-150 \mathrm{mV}$ under UV diode, and $-350 \mathrm{mV}$ under DUV illumination. The estimated error bar is about $\pm 10 \mathrm{mV}$ in all cases. Note, however, that these photovoltages should be taken as approximate values as the light intensity and incident light angle were different for each type of the illumination. Nevertheless, the potential shifts exhibit the same trend.

\section{DISCUSSION}

Although absolute potential values are measured by KPFM, they actually correspond only to relative differences of surface potentials or photovoltages. Only if the potentials are referenced to a known work function, absolute values of the surface work functions or Fermi level energies can be deduced. In all our measurements, the potential of the grounded Ohmic contact $(\mathrm{Ti}+\mathrm{WC})$ did not change during the experiments including various illumination. It evidences that the AFM tip itself is not contributing to the observed photovoltages. It also confirms that this contact can be well used as a reference.

However, there is a large discrepancy in the reported WC work function. Some works indicate 3.6-3.73 eV under 
ultra-high vacuum conditions, ${ }^{14,15}$ other works indicate around $5 \mathrm{eV}{ }^{16,17}$ Our TPYS measurements and Fowler plot analysis provided work function of $4.9 \pm 0.1 \mathrm{eV}$ on our asinserted WC film, i.e., without intentional degassing in vacuum.

By using the guideline in Fig. 2, we can construct the energetic configuration of the O-BDD/WC Schottky junction. Based on the potential difference $(0.4 \mathrm{eV})$ between the WC Ohmic contact and O-BDD surface observed by KPFM in dark, the Fermi level of the exposed O-BDD is then expected to lie around $5.3 \mathrm{eV}$ below the vacuum level. TPYS measurements did not detect ionization energy up to $7.2 \mathrm{eV}$ (applicable detection range limit). Thus, the electron affinity of the wet-chemically oxidized O-BDD must be at least $+1.7 \mathrm{eV}$, in a good agreement with the value reported in the literature. ${ }^{18}$ Assuming this value, the Fermi level at the surface is located $3.6 \mathrm{eV}$ below the conduction band minimum. This is in the energetic region of the diamond surface states. ${ }^{19,20}$ On the other hand, bulk Fermi level in BDD is typically $>4.8 \mathrm{eV}$ below conduction band minimum, ${ }^{21}$ i.e., between energetic level of Boron acceptors and the deep defect states in lowly doped BDD. Thus, the energetic bands as well as the energetic levels of Boron acceptors and related bulk deep defect states are bent downwards at the O-BDD surface.

Because the work function of $\mathrm{WC}(4.9 \mathrm{eV})$ is lower than that of O-BDD $(5.3 \mathrm{eV})$, Schottky junction is formed by holes diffusing from O-BDD to WC Schottky contact. This is evidenced by more positive potential on the floating Schottky WC contact compared to grounded Ohmic WC/Ti contact in the dark as shown in Figs. 3 and 4. Similarly, downward band bending occurs at the junction of O-BDD to Ib substrate. The band banding at the interfaces with both $\mathrm{Ib}$ diamond substrate and WC Schottky contact confine the holes in valence band within O-BDD layer. The corresponding energetic model of the system in equilibrium in the dark assuming the above results and considerations is shown in Fig. 5(a). The KPFM data thus directly corroborate the energetic band configuration in the device which was deduced by Liao et al. based on photocurrent measurements. ${ }^{8}$

Explaining the potential shift under illumination is not that straightforward though. Under the white light illumination, negative shift of surface potential can correspond to electron accumulation such as in the case of organic heterojunction $^{22}$ or electrostatic charging of oxidized intrinsic diamond, ${ }^{23}$ or it can correspond to the downward shift of surface Fermi level and hereby increased work function and accumulation of holes such as in the case of H-terminated intrinsic diamond. ${ }^{24,25}$ To resolve the proper mechanism, we can analyze the situation as follows.

Electrostatic charging or electron accumulation is unlikely in the BDD case as a p-doped material is studied. In addition, Fermi level in Schottky contacts is typically strongly pinned to surface states on oxidized diamond. ${ }^{26}$ Thus, the change of barrier height can be excluded as well. The decrease of potential on Schottky junction under illumination can thus correspond only to the downward shift of Fermi level towards valence band in the BDD epilayer below it. Similar explanation applies to the bare O-BDD. Note that
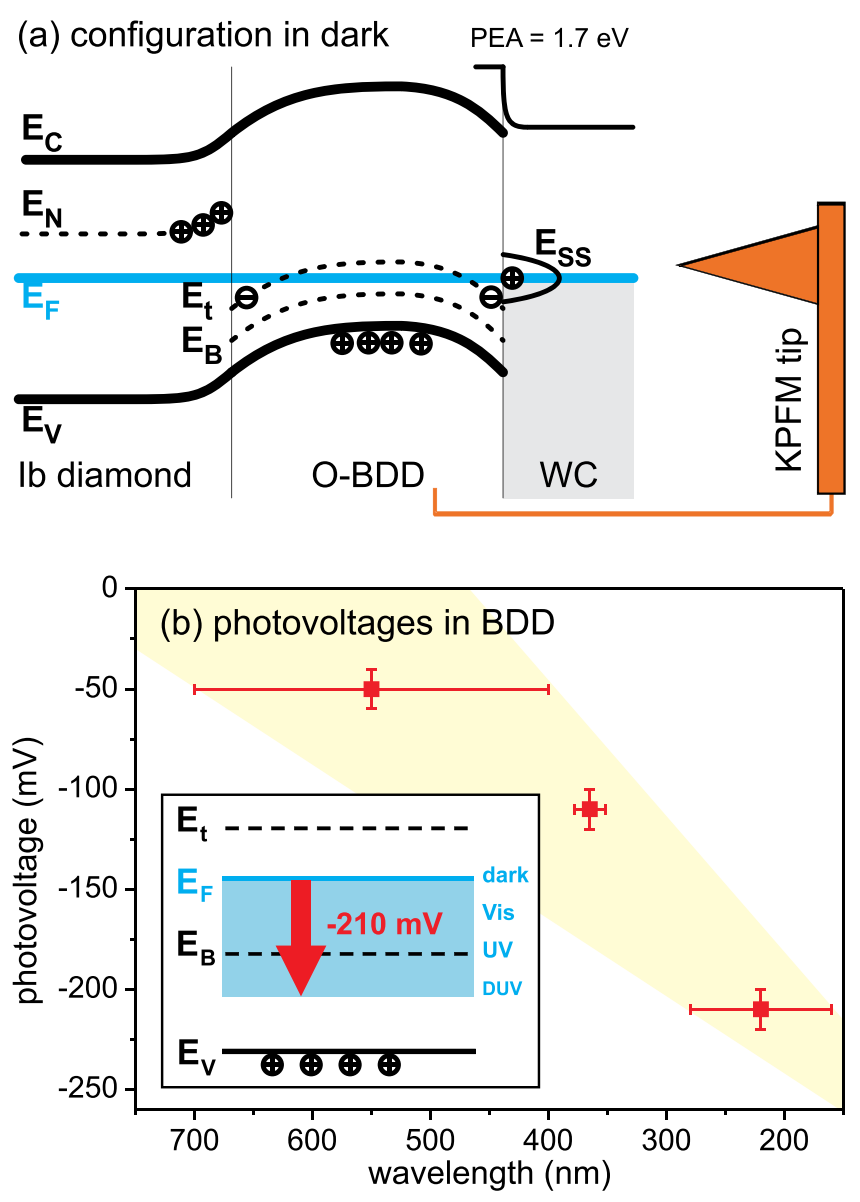

FIG. 5. (a) Schematic diagram showing energetic band configuration and spatial charge regions across BDD Schottky junction in the dark. The KPFM tip is also depicted there to show the unconventional connection of the KPFM to the buried BDD channel that is then measured from top in perpendicular to the involved junctions. (b) Photovoltage as a function of illumination wavelength. The X-error bars indicate band with of the illumination. The inset scheme shows the proposed correlation of the photovoltages with energetic levels and Fermi level shift in the BDD channel. The symbols in both graphs are following: conduction band minimum $\left(\mathrm{E}_{C}\right)$, valence band maximum $\left(\mathrm{E}_{V}\right)$, Fermi level $\left(\mathrm{E}_{F}\right)$, nitrogen related states $\left(\mathrm{E}_{N}\right)$, Boron acceptor level $\left(\mathrm{E}_{B}\right)$, deep level trap states introduced by B-doping $\left(\mathrm{E}_{t}\right)$, surface states on O-BDD $\left(\mathrm{E}_{S S}\right)$, and positive electron affinity (PEA).

the potential of Schottky contact changes under illumination in spite of the contact being a metal. This is because the Schottky contact is electrically floating and semitransparent. Therefore, KPFM detects also opto-electronic effects in the junction(s) and materials below it. ${ }^{9,27}$

The observed photovoltage amplitudes of 50 to $210 \mathrm{mV}$ for white light to DUV illumination are in a good agreement with the range of reported Fermi level position in BDD as a function of doping level. ${ }^{28}$ In general, the doping level controls the number of free charge carriers (holes in the case of $\mathrm{BDD}$ ) in the material. As the number of dopant atoms is fixed here, the Fermi level shift has to correspond to the increased number of holes generated in BDD by illumination. Photovoltages calculated from the KPFM profiles are shown as a function of illumination wavelength in Fig. 5(b). The X-error bars indicate band width of the illumination.

The photovoltage trend evidences that Fermi level moves monotonously towards the BDD valence band and thus more holes accumulate in the BDD channel as the 
illumination energy increases. The model correlating photovoltages with Fermi level shift in the BDD channel is schematically shown as the inset in Fig. 5(b). Considering correlation of acceptor density and bulk Fermi level position in Boron-doped diamond, ${ }^{28}$ the model implies that even small photovoltage variations can lead to orders of magnitude changes in the hole concentration and hence photocurrent depending on the illumination wavelength. For instance, as the reported photocurrent gain ${ }^{6}$ is about 10000 under DUV, the corresponding hole concentration is above $10^{15}$ in that case, even though the BDD layer is doped just unintentionally. Thus, bulk Fermi level moves below Boron acceptor level. The expected shift is about $0.2 \mathrm{eV}^{28}$ This value is in a very good agreement with the amplitude of the observed DUV photovoltage.

Although the above model fits well to all illumination wavelengths, actual mechanisms of the Schottky photodiode function are quite different in the cases of the sub-band and super-band gap illumination.

Under sub-band gap (white light or UV) illumination, holes can be excited to the BDD valence band only from the surface states on O-BDD. The energy is neither enough to excite trapped holes from nitrogen levels in Ib substrate ( $3.8 \mathrm{eV}$ needed) nor to generate transitions above the diamond band gap of $5.5 \mathrm{eV}$. Hence, the positive charge in the O-BDD surface states and the depletion region width of the Schottky junction are reduced. Barrier height is not modified though due to strong Fermi level pinning in the surface states. ${ }^{26}$ When sub-band gap illumination is switched off the holes quickly recombine with the nearby states from which they were excited. Thus, there is no persistent photovoltage and photocurrent.

On the other hand, deep UV (DUV) photons are able to generate electron-hole pairs to diamond valence and conduction bands, which apply for both the Ib diamond substrate and BDD bulk volume. ${ }^{8}$ It has been shown that thicker BDD layer leads to lower photocurrent gain. ${ }^{29}$ Thus, the main contribution must come from the Ib/BDD interface, not from the Schottky junction itself, where the amount of absorbed light is not influenced by the BDD layer thickness.

The DUV illumination intensity at the Ib/BDD interface is obviously strongly reduced by absorption in the BDD layer. However, its contribution to the overall photovoltage effect should be considered as well, as the reported penetration depths in diamond for $220 \mathrm{~nm}$ and $190 \mathrm{~nm}$ photons are $10 \mu \mathrm{m}$ and $1.3 \mu \mathrm{m}$, respectively, ${ }^{30}$ which is much longer than the epitaxial layer thickness $(500 \mathrm{~nm})$, even when considering a slight angle of illumination $\left(\sim 30^{\circ}\right)$ with respect to the sample normal.

Additional holes can be generated from nitrogen states in the Ib substrate (see the scheme in Fig. 5(a)) or from possible defect states at the Ib/BDD interface. ${ }^{31,32}$ However, the KPFM cannot discriminate these origins. Nevertheless, in all cases, the holes are generated to the valence band and follow the potential slope to the BDD layer. Therefore, the DUV illumination leads to large accumulation of holes in BDD. This further shifts the Fermi level down and gives rise to the increasingly negative photovoltage.
When the super-band gap DUV illumination is switched off, some holes may recombine with the nearby defect states as in the case of sub-band gap illumination. However, the majority of excess holes is spatially separated from their original states. Therefore, their recombination rate is low and the photovoltage remains persistent as indeed observed. The work function difference between Ti/WC ohmic contact and WC Schottky contact is large enough to promote diffusion of these holes from BDD towards the Schottky contact. Thus, electrical current can be observed even under zerobias condition. $^{8}$

\section{CONCLUSIONS}

The KPFM data in dark and under illumination lead us to the conclusion that the photovoltage on the electrically floating Schottky contact is directly corresponding to the shift of Fermi level inside the BDD channel below the contact while other contributions are negligible. This unusual relation is most likely enabled by the specific device configuration. In addition, it allowed us to explain how the small photovoltages within $210 \mathrm{mV}$ lead to orders of magnitude difference in photocurrents in such devices. Persistent photovoltage observed in the case of deep UV illumination is in a good agreement with persistent photocurrents, too. We discussed that in spite of monotonous photovoltage trend with illumination, different mechanisms of photovoltage generation under sub-band and super-band gap illumination come into effect. The photovoltage measurements obtained by KPFM under various illumination hence provide direct insight into the opto-electronic function of the diamondbased Schottky photodiodes and similar devices.

\section{ACKNOWLEDGMENTS}

This research was financially supported by the Project Nos. P108/12/G108 (GAČR) and M100101209 (ASCR). The work occurred in frame of the LNSM infrastructure. Additional support by JSPS Invitation Fellowship (S-11064) is gratefully acknowledged.

${ }^{1}$ T. Makino, K. Yoshino, N. Sakai, K. Uchida, S. Koizumi, H. Kato, D. Takeuchi, M. Ogura, K. Oyama, T. Matsumoto, H. Okushi, and S. Yamasaki, Appl. Phys. Lett. 99, 061110 (2011).

${ }^{2}$ Y. Koide, M. Liao, and J. Alvarez, Diamond Relat. Mater. 15, 1962 (2006).

${ }^{3}$ R. McKeag, S. Chan, and R. Jackman, Appl. Phys. Lett. 67, 2117 (1995). ${ }^{4}$ A. BenMoussa, J. Hochedez, U. Schühle, W. Schmutz, K. Haenen, Y. Stockman, A. Soltani, F. Scholze, U. Kroth, V. Mortet, A. Theissen, C. Laubis, M. Richter, S. Koller, J.-M. Defise, and S. Koizumi, Diamond Relat. Mater. 15, 802 (2006).

${ }^{5}$ A. BenMoussa, A. Theissen, F. Scholze, J. Hochedez, U. Schühle, W. Schmulz, K. Haenen, Y. Stockman, A. Soltani, D. McMullin, R. Vest, U. Kroth, C. Laubis, M. Richter, V. Mortet, S. Gissot, V. Delouille, M. Dominique, S. Koller, J. Halain, Z. Remes, R. Petersen, M. D’Olieslaeger, and J.-M. Defise, Nucl. Instrum. Methods Phys. Res., Sect. A 568, 398 (2006).

${ }^{6}$ M. Liao, Y. Koide, and J. Alvarez, Appl. Phys. Lett. 87, 022105 (2005).

${ }^{7}$ Y. Koide, Appl. Surf. Sci. 254, 6268 (2008).

${ }^{8}$ M. Liao, Y. Koide, J. Alvarez, M. Imura, and J.-P. Kleider, Phys. Rev. B 78, 045112 (2008).

${ }^{9}$ B. Rezek, J. Čermák, A. Kromka, M. Ledinský, and J. Kočka, Diamond Relat. Mater. 18, 249 (2009). 
${ }^{10}$ D. Takeuchi, C. Nebel, and S. Yamasaki, Phys. Status Solidi A 203, 3100 (2006).

${ }^{11}$ D. Takeuchi, M. Riedel, J. Ristein, and L. Ley, Phys. Rev. B 68 , 041304(R) (2003).

${ }^{12}$ B. Rezek, C. Sauerer, C. Nebel, M. Stutzmann, J. Ristein, L. Ley, E. Snidero, and P. Bergonzo, Appl. Phys. Lett. 82, 2266 (2003).

${ }^{13}$ C. Fadley, Electron Spectroscopy: Theory, Techniques, and Applications, edited by C. Brundle and A. Baker (Academic Press, London, 1978) p. 15.

${ }^{14}$ W. Tsang, V. Stolojan, S. Wong, J. Linder, B. Sealy, and S. Silva, Rev. Adv. Mater. Sci. 15, 179 (2007).

${ }^{15}$ J. Sun, in Proceedings of the 2008 International Conference on Advanced Infocomm Technology (ACM, New York, NY, 2008), Article No. 81.

${ }^{16}$ Y.-H. Kim, H. Irie, and K. Hashimoto, Appl. Phys. Lett. 92, 182107 (2008).

${ }^{17}$ A. Knizhnik, A. Safonov, I. M. Iskandarova, A. Bagatur'yants, B. Potapkin, L. Fonseca, and M. Stoker, J. Appl. Phys. 99, 084104 (2006).

${ }^{18}$ F. Maier, J. Ristein, and L. Ley, Phys. Rev. B 64, 165411 (2001).

${ }^{19}$ M. Tachiki, Y. Kaibara, Y. Sumikawa, M. Shigeno, H. Kanazawa, T. Banno, K. Song, H. Umezawa, and H. Kawarada, Surf. Sci. 581, 207 (2005).

${ }^{20}$ D. Takeuchi, C. Nebel, and S. Yamasaki, Diamond Relat. Mater. 16, 823 (2007).
${ }^{21}$ M. Geis, J. Twichell, N. Efremow, K. Krohn, and T. Lyszczarz, Appl. Phys. Lett. 68, 2294 (1996).

${ }^{22}$ J. Črmák, B. Rezek, V. Cimrová, A. Fejfar, A. Purkrt, M. Vaněček, and J. Kočka, Thin Solid Films 519, 836 (2010).

${ }^{23}$ E. Verveniotis, A. Kromka, M. Ledinský, J. Čermák, and B. Rezek, Nanoscale Res. Lett. 6, 144 (2011).

${ }^{24}$ B. Rezek, T. Mates, J. Stuchlík, J. Kočka, and A. Stemmer, Appl. Phys. Lett. 83, 1764 (2003).

${ }^{25}$ B. Rezek and C. Nebel, Diamond Relat. Mater. 14, 466 (2005).

${ }^{26}$ D. Takeuchi, S. Yamanaka, H. Watanabe, and H. Okushi, Phys. Status Solidi A 186, 269 (2001).

${ }^{27}$ L. Kronik and Y. Shapira, Surf. Interface Anal. 31, 954 (2001).

${ }^{28}$ C. Bandis and B. Pate, Phys. Rev. B 52, 12056 (1995).

${ }^{29}$ M. Liao, L. Sang, T. Teraji, M. Imura, J. Alvarez, and Y. Koide, Jpn. J. Appl. Phys., Part 1 51, 090115 (2012).

${ }^{30}$ R. Brescia, A. D. Sio, E. Pace, and M. Castex, Diamond Relat. Mater. 13, 938 (2004).

${ }^{31}$ S.-G. Ri, X. Yuan, T. Sekiguchi, N. Tokuda, M. Ogura, H. Okushi, and S. Yamasaki, Diamond Relat. Mater. 17, 489 (2008).

${ }^{32}$ P.-N. Volpe, P. Muret, and F. Omnes, Phys. Status Solidi A 205, 2173 (2008). 